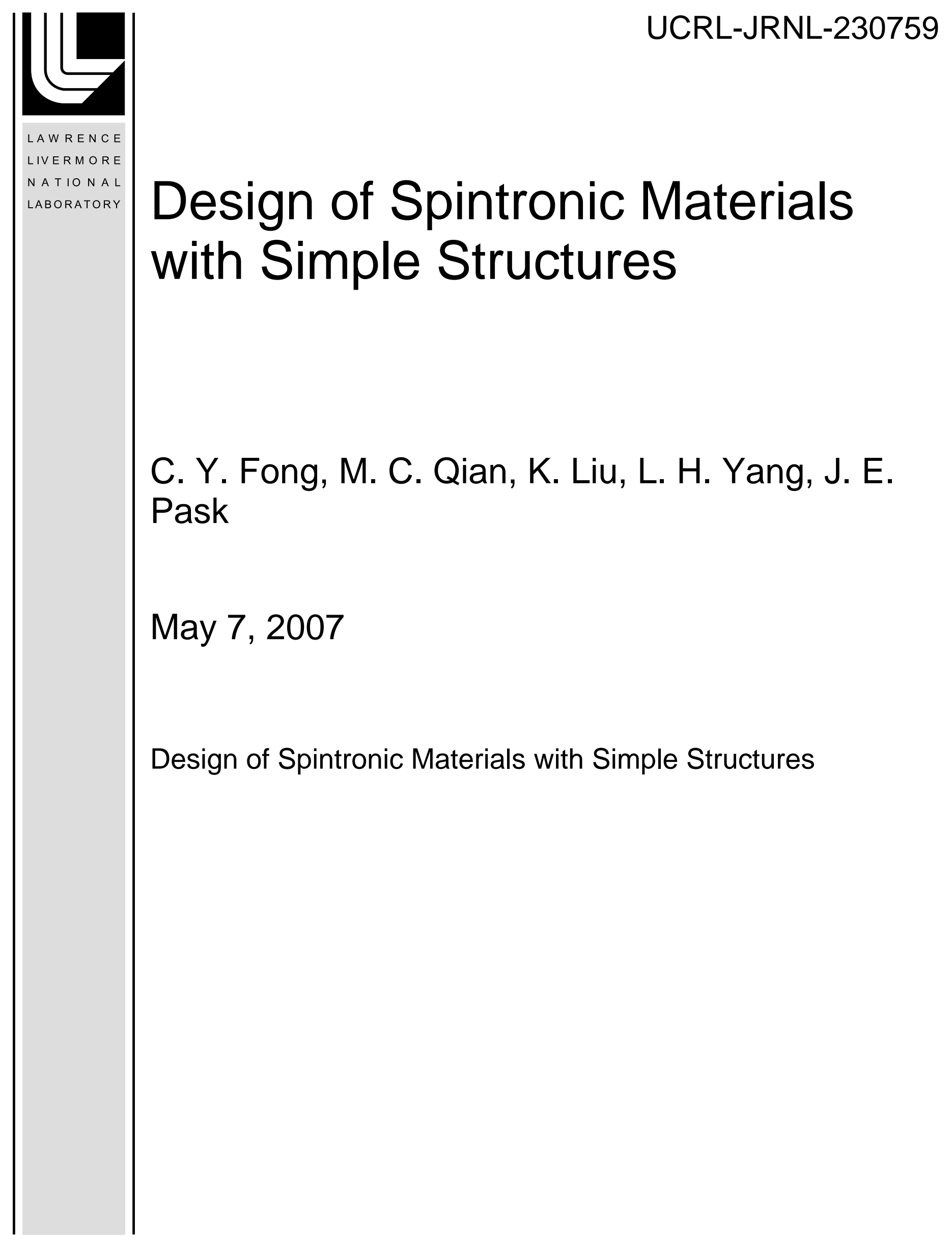


This document was prepared as an account of work sponsored by an agency of the United States Government. Neither the United States Government nor the University of California nor any of their employees, makes any warranty, express or implied, or assumes any legal liability or responsibility for the accuracy, completeness, or usefulness of any information, apparatus, product, or process disclosed, or represents that its use would not infringe privately owned rights. Reference herein to any specific commercial product, process, or service by trade name, trademark, manufacturer, or otherwise, does not necessarily constitute or imply its endorsement, recommendation, or favoring by the United States Government or the University of California. The views and opinions of authors expressed herein do not necessarily state or reflect those of the United States Government or the University of California, and shall not be used for advertising or product endorsement purposes. 


\title{
Design of Spintronic Materials with Simple Structures
}

\author{
C. Y. Fong ${ }^{1 *}$, M. C. Qian ${ }^{1}$, Kai Liu ${ }^{1}$, L. H. Yang ${ }^{2}$, and J. E. Pask ${ }^{2}$ \\ ${ }^{1}$ Department of Physics, University of California, Davis, CA 95616-8677, USA \\ ${ }^{2}$ H-Division, Lawrence Livermore National Laboratory, Livermore, CA 94551 USA
}

RECEIVED DATE (to be automatically inserted after your manuscript is accepted)

* Correspondence should be addressed to e-mail: fong@solid.physics.ucdavis.edu

\begin{abstract}
A brief comparison of conventional electronics and spintronics is given. The key features of half metallic binary compounds with the zincblende structure are presented, using MnAs as an example. We discuss the interactions responsible for the half metallic properties. Special properties of superlattices and a digital ferromagnetic heterostructure incorporating zincblende half metals are also discussed.
\end{abstract}

PACS number(s): 75.50.Cc, 71.15.Mb, 71.20.-b 


\section{Introduction}

Since the discovery of giant magnetoresistance (GMR) in $1988^{1}$, there has been tremendous interest in magneto-electronics because of the potential for innovative devices in which the electron spin is employed for information storage, transmission, and manipulation. ${ }^{2-6}$ However, much of the high-tech industry is based on semiconductors, which have seen significant advances in nanofabrication and device integration. It is thus desirable to develop magneto-electronics in a way that takes advantage of these mature technologies. For this purpose, a new terminology "spintronics" has been developed to describe devices that utilize electron spin, or both charge and spin, in their basic operation and incorporate these into semiconductor technologies. We compare and contrast spintronics and conventional electronics in Table 1.

Currently, metal-based magneto-electronic devices in the form of heterostructures, such as $\mathrm{Co}, \mathrm{Ni}$, and $\mathrm{Fe}$ in thin film form, have already found applications in ultra-high density magnetic recording ${ }^{8}$ as GMR sensors, ${ }^{9-10}$ in prototype magnetic random access memory (MRAM), ${ }^{11-13}$ and in spin-current switches. ${ }^{14-15}$ These devices utilize ordinary magnetic materials that have a spin imbalance, or finite electronic spin polarization $P$, at the Fermi energy $E_{F}$. Information, for example, can be encoded based on the finite resistance change associated with the spin-dependent electronic transport through these magnetic heterostructures.

With respect to the sensitivity of such magnetoresistance (MR) based devices, there is another attractive class of materials, the so called "half metals", which should prove markedly superior to the conventional metal based superlattices. Half metals have one spin channel which is metallic while the other is semiconducting. The density of states (DOS) of a typical half metal, zincblende CrAs, ${ }^{16}$ is shown in Fig. 1, in which states of the majority-spin channel are occupied up to $E_{F}$, where those of the minority channel show a gap.

According to the formulation of Julliere ${ }^{17}$, the MR relates to the polarization $P$ at $E_{F}$ as 
$\mathrm{MR}=2 P^{2} /\left(1-P^{2}\right)$

where

$P=\left(\mathrm{DOS}_{\uparrow}-\mathrm{DOS}_{\downarrow}\right) /\left(\mathrm{DOS}_{\uparrow}+\mathrm{DOS}_{\downarrow}\right)$

$P=1(P$ of iron is 0.45$)$ for zincblende CrAs and the corresponding MR $\rightarrow \infty$. Therefore, half metals are ideal candidates for MR based devices: information storage, spin filters, detectors, sensors, and the like. MRAM can be made from a half metallic superlattice arrangement of digital ferromagnetic heterostructure (DFH). ${ }^{18}$ A DFH is a semiconductor, such as GaAs or $\mathrm{Si}, \delta$-doped with a layer of a transition metal element. Half metals can also be used for polarized light sources and optoelectronic devices.

Before 2000, two main types of half metallic compounds were known: the Heusler alloys (NiMnSb, $\mathrm{Ni}_{2} \mathrm{MnSb}, \mathrm{Co}_{2} \mathrm{MnSi}$, etc. $)^{19}$ and oxides $\left(\mathrm{Fe}_{3} \mathrm{O}_{4}, \mathrm{CrO}_{2}\right.$, etc. $){ }^{5}$ Unfortunately, these compounds presented a number of problems in practice. ${ }^{20}$ For the Heusler alloys, there are several atomic species in the unit cell and the complex atomic arrangements (Fig. 2) and difficulties controlling defects and stoichiometry have impeded progress. In NiMnSb, spin flip transitions destroy half metallicity at temperature $T^{*}=88 \mathrm{~K}^{21}$, far below $T_{c}$ and room temperature. Similar difficulties are present in the oxides. In $\mathrm{CrO}_{2}$, multiple crystalline phases can be formed with the same stoichiometry due to the complex structure. The rutile structure is one such half metallic phase, ${ }^{5}$ for example. Furthermore, close to $100 \%$ spin polarization is achieved at low temperature but disappears at room temperature. ${ }^{22}$ The common factor in the difficulties associated with both types of half metals is the complex structure.

In 2000, Akinaga et al. ${ }^{23}$ predicted using first principles calculations that CrAs in the simple zincblende $(\mathrm{ZB})$ structure is a half metal (HM), and then grew it in thin film form on a GaAs substrate. The material has several notable features. $T_{c}$ is estimated to be above $480 \mathrm{~K}$. The simple $\mathrm{ZB}$ structure eliminates the stoichiometry problem and should improve transport properties (by virtue of lacking defects). And from the calculated density of states, as shown in Fig. 1, $P=1$, i.e., 100\% polarization at 
the Fermi level. At about the same time, H. Ohno's group succeeded in growing a monolayer of $\mathrm{CrSb}$ in $\mathrm{GaSb}^{24}$ The significant desirable properties of ZB CrAs stimulate us to design new HM compounds with the ZB structure.

In Section II, we briefly discuss the method of calculations. Results and discussion are presented in Sec. III, and we summarize in Sec. IV.

\section{Method of Calculations}

We used the planewave pseudopotential method, as implemented in $\mathrm{VASP}^{25}$, a first-principles code based on density functional theory. Ultrasoft pseudopotentials for the elements were employed using standard valence configurations: e.g., $\mathrm{Cr}, 3 d^{5} 4 s^{1}$. The Perdew 91 version of the generalized gradient approximation, $\mathrm{GGA}^{26}$, was used for exchange and correlation.

\section{Results and discussion}

\section{A. Half metals with the zincblende structure}

We present the designed half metallic compounds with the $\mathrm{ZB}$ structure $^{16}$ in Table 2 , and in Table 3 list related compounds explored by other research groups. In performing the calculations, we confirmed first the results for CrAs obtained by Akinaga et al., ${ }^{23}$ and then proceeded to explore other transition-metal pnictide and carbide compounds. In Ref. 16, we also suggested well matched substrates for each of the designed compounds to grow on.

\section{B. Features of a half metal with the zincblende structure}

We use MnAs as a prototype to demonstrate the key features of a half metal with the ZB structure.

1. Density of states and magnetic moment

Figure 3 shows the density of states of MnAs as a function of energy. It is clearly half metallic: The up (majority) spin states are metallic, with a significant DOS at $E_{F}$. The down (minority) spin states 
are semiconducting. The magnetic moment $M$ (Table 2) is $4.0 \mu_{\mathrm{B}} /$ unit-cell where $\mu_{\mathrm{B}}$ is the Bohr magneton. This value of $M$ can be understood in terms of (i) the atomic configurations, (ii) the charge transfer process, and (iii) Hund's first rule. Mn has seven valence electrons, $3 d^{5} 4 s^{2}$, and As has five valence electrons, $4 s^{2} 4 p^{3}$. Three of the valence electrons of the Mn atom are transferred to the As atom to form $p$ - $d$ bonding states. There are then four electrons left at the Mn atomic site. Hund's first rule requires the spins of the four electrons to align. Consequently, $M=4.0 \mu_{\mathrm{B}} /$ unit-cell.

The necessarily integer value of $M$, characteristic of half metals, can be readily understood by counting the electrons in the semiconducting channel. The total number of electrons/unit cell, $N_{\mathrm{t}}$, is an integer. The minority-spin channel is semiconducting, and so also contains an integer number of electrons $N_{\downarrow}$. The number of electrons in the majority-spin channel $N_{\uparrow}$ is then necessarily an integer also:

$N_{\uparrow}=N_{\mathrm{t}}-N_{\downarrow}$

$M=\left(N \uparrow-N_{\downarrow}\right) \mu_{\mathrm{B}}$

The integer magnetic moment/unit cell is a necessary condition for a HM. This number can be deduced from a measurement of the saturation magnetization. ${ }^{31}$

2. Interactions for half metallicity

There are three interactions which combine to cause a sample to exhibit half metallic properties. We start with the valence electron configurations for the two atoms: $3 d^{5} 4 s^{2}$ for Mn and $4 s^{2} 4 p^{3}$ for As.

a) The crystal field effect due to the tetrahedral symmetry at the Mn atom splits the $d$-states. The five-fold degenerate atomic $d$-manifold splits into a three-fold $\left(t_{2 \mathrm{~g}}\right)$ and a two-fold $\left(e_{\mathrm{g}}\right)$ with a gap in between. These latter states form the $\Gamma_{12}$ non-bonding states in the ZB structure. 
b) $p$ - $d$ hybridization causes the $t_{2 \mathrm{~g}}$ states and the $p$-states of the As atom to form bonding and antibonding states separated by a gap. This is the major gap. Figure 4 shows how one of the $d$-orbitals of the Mn atom in the ZB structure can hybridize with the $p$-orbital of the As atom. Figure 5 summarizes schematically the above two interactions.

c) The exchange interaction (molecular field) determines the relative energy of the spin-up and spin-down states.

The three interactions are manifested in the band structure. In Fig. 6, we show the band structure of ZB MnAs in the majority- and minority-spin channels. For interaction a), we use the splitting between the lowest triply degenerate $\left(\Gamma_{15}\right)$ and the doubly degenerate $\left(\Gamma_{12}\right)$ states at the $\Gamma$ point $(k=0)$ to characterize the crystal field effect. The splitting of the spin-up states is $0.94 \mathrm{eV}$. For interaction b), the energy difference of the two lowest-energy triply degenerate states $\left(\Gamma_{15}\right)$ at the $\Gamma$ point characterizes the $p$ - $d$ hybridization. The gap is $6.0 \mathrm{eV}$ (spin-up states). For interaction c), we use the energy difference between the lowest triply degenerate $\left(\Gamma_{15}\right)$ states in each spin channel to characterize the exchange interaction. The shift is $1.86 \mathrm{eV}$. In Fig. 7, the charge density of the bonding states $\left(\Gamma_{15}\right)$ along the MnAs chain is shown in the left panel. The bond charge between the Mn and As atoms can be easily identified. The charge density of the nonbonding states $\left(\Gamma_{12}\right)$ along the Mn-As chain is shown in the right panel. No bond charge is seen.

\section{C. $\mathrm{MnC}$}

In all the pnictides, the majority-spin $(\uparrow)$ states show metallic behavior while the minority-spin $(\downarrow)$ channel exhibits semiconducting properties. In $\mathrm{MnC}$, it is the opposite even though the magnetic moment per unit cell in all these cases is determined by the $\uparrow$ states. An interesting consequence will be discussed below. The equilibrium lattice constant ${ }^{29}$ of $\mathrm{ZB} \mathrm{MnC}$ is $4.39 \AA$, as shown in lower part of Fig. 8. The upper part of the figure shows its interesting half metallic properties. For lattice constant $a$ 
greater than $5.0 \AA$, the half metallic properties are qualitatively similar to MnAs. A new half-metallic phase is predicted for lattice constant between $3.6 \AA$ and $4.28 \AA$.

The DOS at $a=4.20 \AA$ is shown in Fig. 9. The majority-spin states are semiconducting while the minority-spin states are metallic. As shown in Fig. 8, the magnetic moment is $1.0 \mu_{\mathrm{B}}$. This value can be understood as follows: The valence electron configuration of the Mn atom is $2 d^{5} 4 s^{2}$ and that of the C atom is $2 s^{2} 2 p^{2}$. Four of the seven Mn electrons are transferred to the $\mathrm{C}$ atom to form bonding states, leaving three electrons at the Mn site. For larger lattice constant $a=5.0 \AA, M=3.0 \mu_{\mathrm{B}}$. In this case, the spins of the three electrons left at the Mn site align themselves to reduce the Coulomb repulsion, consistent with Hund's first rule. The electrons avoid one another due to the Pauli principle. At the smaller lattice constant, however, the volume around each atom is proportionally smaller and interactions with neighboring atoms are stronger. One of the three electrons flips its spin. Consequently, the magnetic moment collapses to $1.0 \mu_{\mathrm{B}}$. The half metallic behavior of $\mathrm{MnC}$ at the smaller lattice constant has an interesting physical implication. Recall that the majority (up) spin channel is semiconducting, with localized charge, and the magnetic moment of the sample is determined by this channel. Therefore, there are periodically arranged localized magnetic moments at the Mn sites. The minority (down) spin channel is metallic, so that the electrons in this channel wander in the crystal and feel the local magnetic moments. The low temperature transport properties should show interesting characteristics. $^{29}$

\section{Superlattices}

${\underline{\mathrm{MnC} / \mathrm{SiC}^{30}}}^{30}$ We found that only $(\mathrm{MnC})_{1}(\mathrm{SiC})_{3}$ and $(\mathrm{MnC})_{1}(\mathrm{SiC})_{5}$ are half metallic and expect that they are difficult to grow. $\mathrm{SiC}$ is a very hard material. In these model superlattices, we are modeling $\mathrm{MnC}$ grown on $\mathrm{SiC}$ which serves as a substrate. If they can be grown, these superlattices can be integrated into $\mathrm{SiC}$ and $\mathrm{C}$ technologies and can possibly be used in high-pressure, high-temperature environments. 
${\underline{\mathrm{CrAs}} / \mathrm{MnAs}^{31}}^{31}$ This type of superlattice is made of CrAs and MnAs. Two superlattices were designed: $(\mathrm{CrAs})_{1}(\mathrm{MnAs})_{1}$ and $(\mathrm{CrAs})_{2}(\mathrm{MnAs})_{2}$. They both show half metallic properties. From Table 2, GaAs can serve as a substrate for these superlattices to be grown on. Therefore, they can be integrated into GaAs technologies.

$\underline{\mathrm{GaAs} / \mathrm{MnAs} /(\mathrm{CrAs})_{2}} \underline{(\mathrm{GaAs})}_{2}{ }_{2}^{32}:$ This superlattice can be thought of as MnAs and CrAs capped with GaAs. It is half metallic. In addition, it shows ballistic transport. The model is shown in Fig. 10. The $\mathbf{x}$-axis is along [110] of the cubic cell. It has GaAs at the bottom and top. In between, it consists of one layer of MnAs and two layers of CrAs. Figure 11 shows (a) the total charge density of the majorityspin channel, (b) the total charge density of the minority-spin channel, and (c) the charge density of the unoccupied states within $0.3 \mathrm{eV}$ above $E_{F}$. This last figure provides evidence that the charge density corresponding to the conduction states near $E_{F}$ extends from left to right, providing a possible ballistic channel in the z-direction. More evidence of ballistic transport in this superlattice is shown in Fig. 12, where the ballistic conductance vs. energy is calculated. At $E_{F}$, only electrons having up-spin polarization contribute to the conductance. The resultant current is $100 \%$ polarized. This kind of superlattice could be used as a spin filter.

\section{E. Digital Ferromagnetic Heterostructure (DFH)}

In practice, it can be difficult to grow half metals in the ZB structure because the $\mathrm{ZB}$ structure is not the ground state. The NiAs structure is the ground state for the pnictides. But the pnictides in this structure are not half metals. Among all the semiconductors, Si technologies are the most mature including growth of alloys with $\mathrm{Si}$. The most promising materials for growth in the near term, therefore, are those with the most Si. DFHs are one possibility. The model of the $\mathrm{DFH}^{33}$ is shown in Fig. 13. It consists of 31 layers of $\mathrm{Si}$ and one layer of $\mathrm{Mn}$. We first relax the atoms. The results of relaxed and unrelaxed cases are compared in Table 4. The gap in the minority-spin states is $0.21 \mathrm{eV}$. This value is underestimated because the GGA is used. To further investigate the electronic properties of this DFH, we plot the Fermi surface in a two dimensional section of the Brillouin zone (BZ), as shown in Fig. 
14(b). A closed Fermi energy contour around the $\Gamma$ point $(k=0)$, the center of the two dimensional $\left(k_{\mathrm{x}^{-}}\right.$ $\left.k_{\mathrm{y}}\right) \mathrm{BZ}$, is formed by the lowest-energy unfilled impurity states - hole surface (see band structure below). At the corners of the BZ, there are electron pockets formed by higher-energy filled impurity states.

The band structure of the DFH is shown in Fig. 15. The impurity states are shown as thicker curves. Near the $\Gamma$ point, there are states above the Fermi energy (band labeled as 1 ) forming the hole surface in Fig. 14(b). The states near the point $\mathrm{R}$ at the zone edge are filled electron states. They are labeled as 2 and 3. They form the electron Fermi surface around $\mathrm{R}$. The point $\mathrm{Z}$ is along the $k_{\mathrm{z}}$ direction and is at the zone boundary. The bands along this direction are relatively flat as compared to the other two directions. Figure 15(b) shows the band structure of the minority-spin states of the DFH. This channel is semiconducting, with a gap of $0.21 \mathrm{eV}$ at $E_{F}$. An important feature is that the Fermi energy is located close to the top of the valence bands.

Recall that one of the half metallic (HM) Heusler alloys (HA), NiMnSb, loses its HM properties at $T^{*}$ well below room temperature. The low $T^{*}$ can be understood in terms of the schematic DOS diagram shown in Fig. 16. The left side corresponds to the majority-spin $\operatorname{DOS}, D \uparrow(E)$ and the right side corresponds to the minority, $D_{\downarrow}(E)$. The $E_{F}$ of the HA is near the bottom of the conduction bands (at the upper end of $\delta$, not shown in the figure). Thermally excited spin flip transitions for electrons with energy at $E_{F}$ to the bottom of the spin-down conduction band will shift the $E_{F}$ to align the spin-up and spin-down states. Thus, the half metallic properties will be destroyed.

For the Si-based DFH, the $E_{F}$ is just above the top of the valence band (VBM), indicated by the solid line labeled by $E_{F}$. The spin flip transition marked by $\delta(0.21 \mathrm{eV})$ is the same as in the HA but the energy difference is about 10 times larger than the thermal energy. This renders the transitions highly improbable. The other spin flip transition can involve states from the minority-spin states at the VBM making transitions to the majority-spin states at the $E_{F}$. However, the down-spin valence electrons are bonded and close to the Si atoms (forming the $p$ - $d$ bonding states). These spin flip transitions are 
suppressed by the matrix elements which depend on the overlap between the initial and the final states.

Consequently, spin flip transitions are highly improbable in our newly designed Si-based half metal, and so it is robust with respect to them.

\section{Summary}

Based on first-principles, density-functional calculations, we have designed half metallic materials with the simple ZB structure, related superlattices, and a Si-based digital ferromagnetic heterostructure. Most of the ZB half metallic crystals show metallic properties in the spin-up channel and semiconducting behavior in the spin-down. The magnetic moment of any of these samples can be readily understood in terms of charge transfer and Hund's first rule.

The up- and down-spin states play opposite roles in $\mathrm{MnC}$ with its lattice constant less than $4.28 \AA$. This unusual HM property for MnC can be understood in terms of the volume effect and Hund's first rule, and should show interesting low temperature transport properties. Only one layer of $\mathrm{MnC}$ can be grown on $\mathrm{SiC}$ in the form of a superlattice. It is possible to make HM superlattices composed of MnAs and CrAs. In addition, we designed a superlattice composed of MnAs and CrAs capped by GaAs to exhibit ballistic transport. A Si-based digital ferromagnetic heterostructure (DFH) has been presented. It exhibits two-dimensional half metallicity. The low $T^{*}$ in Heusler alloys associated with spin flip transitions will not occur in this DFH. This DFH thus has excellent prospects for device applications.

\section{Acknowledgments}

Work at UC Davis was supported in part by NSF with Grant No. ECS-0225007, SDSC, and the Alfred P. Sloan Foundation (K.L.). Work at LLNL was performed under the auspices of the U.S. Department of Energy by the University of California Lawrence Livermore National Laboratory (LLNL) under contract No. W-7405-Eng.-48. 


\section{References}

1. M. N. Baibich, J. M. Broto, A. Fert, F. Nguyen Van Dau, F. Petroff, P. Eitenne, G. Creuzet, A. Friederich, and J. Chazelas, Phys. Rev. Lett. 61, 2472 (1988).

2. See e.g., Physics Today, 48, (4) (1995).

3. G. A. Prinz, Science 282, 1660 (1998).

4. D. D. Awschalom, M. E. Flatte, and N. Samarth, Sci. Am. 286, 66 (2002).

5. See special issue, MRS Bulletin, October (2003); S. P. Lewis, P. B. Allen, T. Sasaki, Phys. Rev. B, 55, 10253 (1997); Y. S. Dedkov, U. Rudiger, G. Guntherodt, Phys. Rev. B, 65, 064417 (2002).

6. Zutic, J. Fabian, and S. Das Sarma, Rev. Mod. Phys. 76, 323 (2004).

7. S. A. Wolf, D. D. Awschalom, R. A. Buhrman, J. M. Daughton, S. von Molnar, M. L. Roukes, A. Y. Chtchelkanova, and D. M. Treger, Science 294, 1488 (2001).

8. C. Ross, Ann. Rev. Mater. Res. 31, 203 (2001).

9. $\quad$ S. S. P. Parkin, R. Bhadra, and K. P. Roche, Phys. Rev. Lett. 66, 2152 (1991).

10. B. Dieny, V. S. Speriosu, S. S. P. Parkin, B. A. Gurney, D. R. Wilhoit, and D. Mauri, Phys. Rev. B 43, 1297 (1991).

11. J. M. Daughton, Thin Solid Films 216, 162 (1992).

12. L. Savtchenko, B. N. Engel, N. D. Rizzo, M. F. Deherrera, and J. A. Janesky, US Patent, 6,545,906 B1 (2003).

13. K. Bussmann, G. A. Prinz, S. F. Cheng, and D. Wang, Appl. Phys. Lett. 75, 2476 (1999).

14. J. C. Slonczewski, J. Magn. Magn. Mater. 159, L1 (1996).

15. J. A. Katine, F. J. Albert, R. A. Buhrman, E. B. Myers, and D. C. Ralph, Phys. Rev. Lett. 84, 3149 (2000).

16. J. E. Pask, L. H. Yang, C. Y. Fong, W. E. Pickett, and S. Dag, Phys. Rev. B 67, 224420 (2003).

17. M. Julliere, Phys. Lett. A 54, 225 (1975).

18. J. A. Schuller, E. Johnson-Halperin, C. S. Gallinat, H. Knotz, A. C. Gossard, and D. D. Awschalom, J. Appl. Phys. 95, 4922 (2004).

19. R. A. deGroot, F. M. Mueller, P. G. Vanengen, and K. H. J. Buschow, Phys. Rev. Lett. 50, 2024 (1983).

20. M. Ziese, Rep. Prog. Phys. 65, 143 (2002).

21. C. Hordequin, D. Ristoiu, L. Ranno, and J. Pierre, Eur. Phys. J. B 16, 287 (2000).

22. Y. Ji, G. J. Strijkers, F. Y. Yang, C. L. Chien, J. M. Byers, A. Anguelouch, G. Xiao, and A. Gupta, Phys. Rev. Lett. 86, 5585 (2001). 
23. H. Akinaga, T. Manago, and M. Shirai, Jpn. J. Appl. Phys. Lett. 39, L1118 (2000).

24. J. H. Zhao, F. Matsukura, K. Takamura, E. Abe, D. Chiba, and H. Ohno, Appl. Phys. Lett. 79, $2776(2001)$.

25. G. Kresse and J. Hafner, J. Phys.: Condens. Matter 6, 8245 (1994).

26. J. P. Perdew, K. Burke, and M. Ernzerhof, Phys. Rev. Lett. 77, 3865 (1996).

27. I. Galanakis and P. Mavropoulos, Phys. Rev. B 67, 104417 (2003).

28. W. H. Xie, Y. Q. Xu, B. G. Liu, and D. G. Pettifor, Phys. Rev. Lett. 91, 037204 (2003).

29. M. C. Qian, C. Y. Fong, and L. H. Yang, Phys. Rev. B 70, 052404 (2004).

30. C. Y. Fong and M. C. Qian, J. Phys. Condens. Matter 16, S5669 (2004).

31. C. Y. Fong, M. C. Qian, J. E. Pask, L. H. Yang, and S. Dag, Appl. Phys. Lett. 84, 239 (2004).

32. M. C. Qian, C. Y. Fong, W. E. Pickett, J. E. Pask, L. H. Yang, and S. Dag, Phys. Rev. B 71, 012414 (2005).

33. M. C. Qian, C. Y. Fong, K, Liu, W. E. Pickett, L. H. Yang, and J. E. Pask, Phys. Rev. Lett. 96, 027211 (2006). 
Table 1. Comparison of conventional electronics and spintronics.

\begin{tabular}{|l|l|l|}
\hline & Electronics & Spintronics \\
\hline Carrier & Charge & Spin or both charge and spin \\
\hline $\begin{array}{l}\text { Speed of transferring } \\
\text { information and power } \\
\text { dissipation }\end{array}$ & $\begin{array}{l}\text { Limited in speed, } \\
\text { Significant power dissipation }\end{array}$ & $\begin{array}{l}\text { Much higher speed, } \\
\text { Lower power dissipation }\end{array}$ \\
\hline Information storage & 0 and 1 bits & $\begin{array}{l}\text { More possibilities: } \\
\text { a. Magnetoresistance (0,1), and b. Qbits } \\
\text { (using spin information) }\end{array}$ \\
\hline
\end{tabular}


Table 2. Predicted half metals with zincblende structure. $a_{\mathrm{o}}$ is the equilibrium lattice constant and $a_{\mathrm{HM}}$ is the lattice constant at which the compound begins to show half metallic properties. $\mu_{\mathrm{B}}$ is the Bohr magneton. $(\uparrow)$ denotes spin up, $(\downarrow)$ denotes spin down.

\begin{tabular}{|l|l|l|l|l|l|}
\hline Compound & $a_{o}(\AA)$ & $a_{\mathrm{HM}}(\AA)$ & $\begin{array}{l}\text { DOS at } E_{F} \\
(\text { states/eV-unit cell })\end{array}$ & Gap $(\mathrm{eV})$ & $\mathrm{M}\left(\mu_{\mathrm{B}}\right)$ \\
\hline $\mathrm{CrAs}$ & 5.66 & 5.66 & $0.85(\uparrow)$ & $1.85(\downarrow)$ & 3.0 \\
\hline $\mathrm{CrP}$ & 5.42 & 5.48 & $0.82(\uparrow)$ & $1.86(\downarrow)$ & 3.0 \\
\hline $\mathrm{CrSb}$ & 6.14 & 6.14 & $0.87(\uparrow)$ & $1.54(\downarrow)$ & 3.0 \\
\hline $\mathrm{MnAs}$ & 5.74 & 5.77 & $0.77(\uparrow)$ & $1.70(\downarrow)$ & 4.0 \\
\hline $\mathrm{MnSb}$ & 6.21 & 6.21 & $0.79(\uparrow)$ & $1.40(\downarrow)$ & 4.0 \\
\hline $\mathrm{MnC}$ & 4.39 & 4.20 & $1.24(\downarrow)$ & $0.90(\uparrow)$ & 1.0 \\
\hline $\mathrm{TiSb}$ & 6.29 & 6.29 & $2.32(\uparrow)$ & $1.40(\downarrow)$ & 1.0 \\
\hline
\end{tabular}


Table 3. Predictions of half metals with zincblende structure by two other groups. Numbers in parentheses are lattice constants.

\begin{tabular}{|l|l|l|}
\hline Research group & Substrate & Compounds \\
\hline Galanakis, Mavropoulos ${ }^{27}$ & GaAs (5.65 $\AA)$. & $\begin{array}{l}\text { VAs }(5.54 \AA), \mathrm{VN}(4.21 \AA), \mathrm{VP}(5.27 \AA), \\
\mathrm{CrP}(5.19 \AA), \mathrm{CrAs}(5.52 \AA)\end{array}$ \\
\hline Xie, Xu, and Liu ${ }^{28}$ & & $\begin{array}{l}\text { CrAs, CrSb, CrSe, MnAs, MnSb, MnBi, VTeCrSe, } \\
\text { MnAs, MnSb, MnBi, VTe. }\end{array}$ \\
\hline
\end{tabular}


Table 4. Comparison of the properties of unrelaxed and relaxed model Mn/Si-DFH. "spin-up" and "spin-down" indicate the DOSs of the spin-up and spin-down channels.

\begin{tabular}{|c|c|c|c|c|c|}
\hline Lattice Relaxation & spin-up & $\begin{array}{l}\text { S at } E_{F} \\
V \text {-unit cell) } \\
\text { spin-down }\end{array}$ & $\begin{array}{l}\text { Magnetic moment } \\
\qquad\left(\mu_{\mathrm{B}}\right)\end{array}$ & Total energy $(\mathrm{eV})$ & Gap (eV) \\
\hline Without & $\begin{array}{l}5.06 \\
7.40\end{array}$ & $\begin{array}{l}0.00 \\
7.40\end{array}$ & $\begin{array}{ll}12.0 \mathrm{FM} \\
0.0 \mathrm{AFM}\end{array}$ & $\begin{array}{l}0.0 \text { ( Reference) } \\
0.435\end{array}$ & 0.16 \\
\hline With & $\begin{array}{l}5.95 \\
7.54\end{array}$ & $\begin{array}{l}0.00 \\
7.54\end{array}$ & $\begin{array}{l}12.0 \mathrm{FM} \\
0.0 \text { AFM }\end{array}$ & $\begin{array}{l}-0.116 \\
0.267\end{array}$ & 0.21 \\
\hline
\end{tabular}


Figure 1. Total density of states for the CrAs with the zincblende structure.

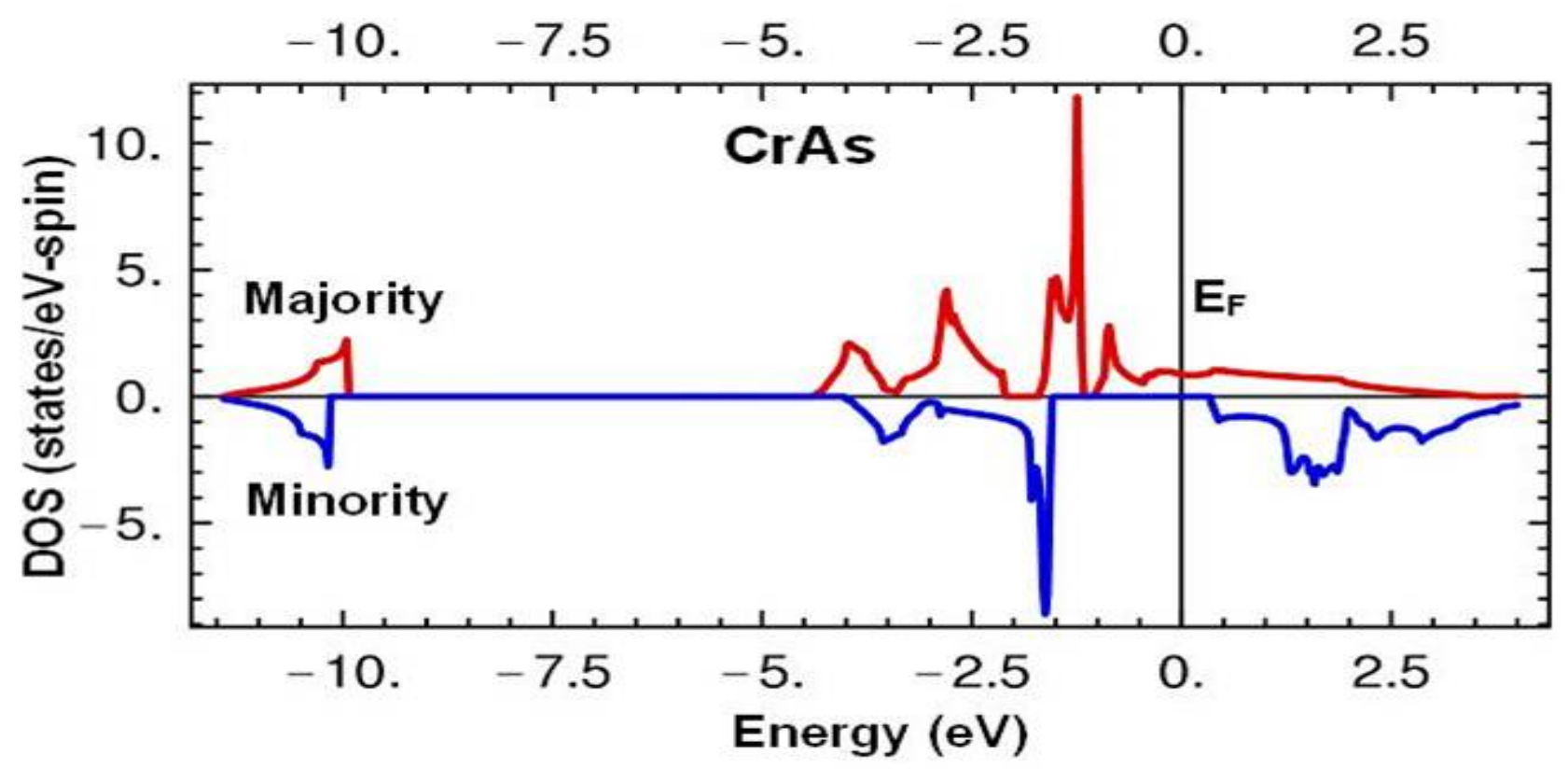


Figure 2. Schematic projection diagram of the structure of a typical Heusler alloy, $\mathrm{A}_{2} \mathrm{BC}$. $\mathrm{a}$ is the lattice constant. Green ellipses are C atoms located at corners and face centers of a cube. Red ellipses are B atoms at line centers of a cube. Gray and blue ellipses are A atoms, which form another cube around the central C.

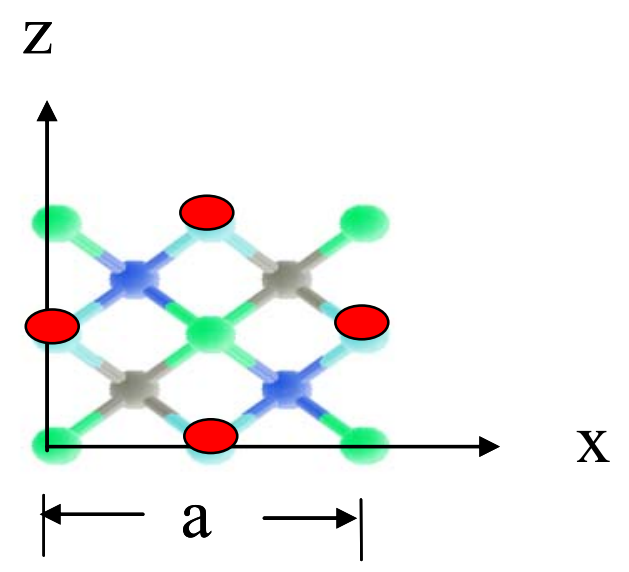


Figure 3. Total density of states for MnAs with the zincblende structure.

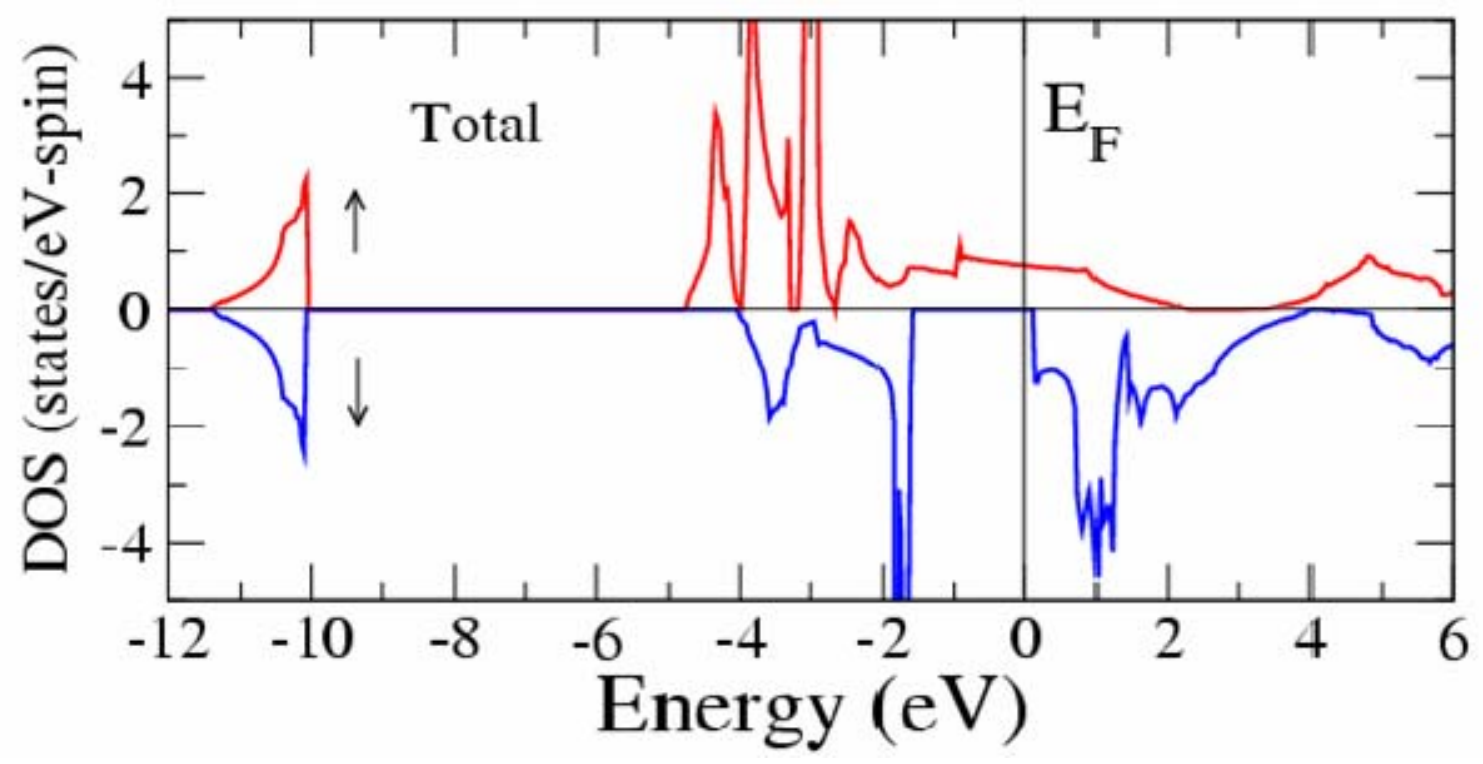


Figure 4. (a) $p$ - $d$ hybridization. $d_{\mathrm{xy}}$ is one of the triply degenerate $d$ states. (b) Key elements of the zincblende structure.
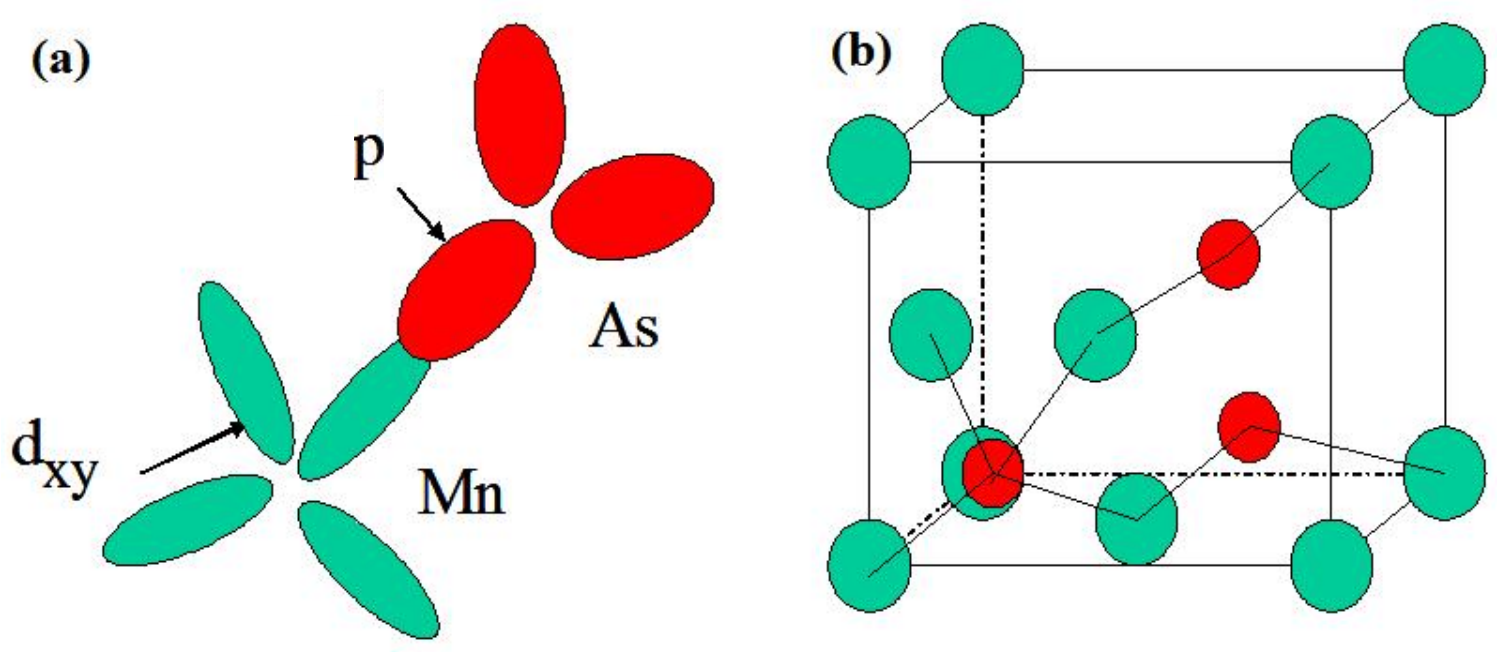
Figure 5. Schematic diagram showing crystal field and hybridization effects. The superscripts indicate the occupancy of the state.

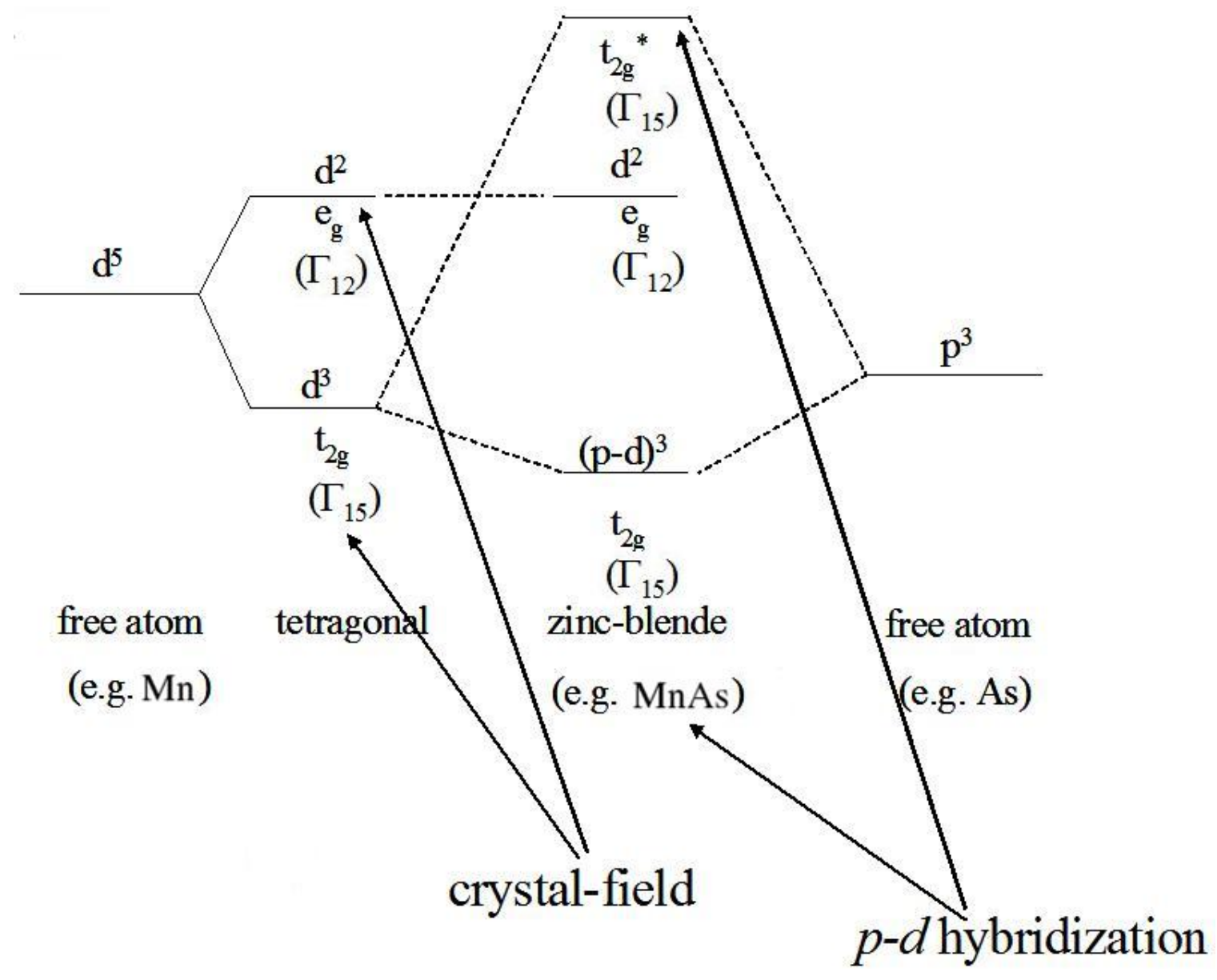


Figure 6. Band structure of zincblende MnAs.

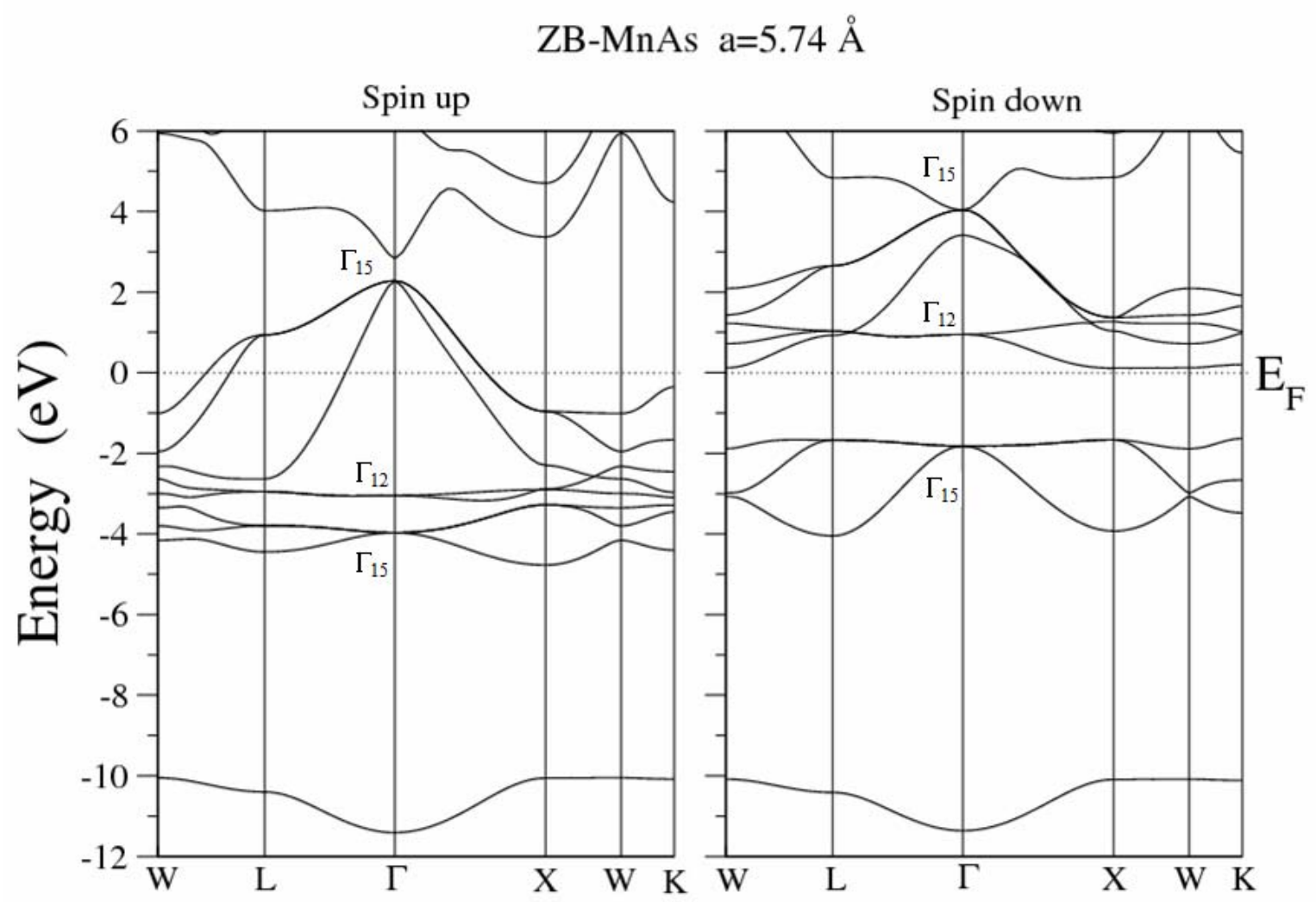


Figure 7. Charge densities of (a) bonding $\Gamma_{15}$ state and (b) nonbonding $\Gamma_{12}$ state in the majority-spin channel along the Mn-As chain.

(a)

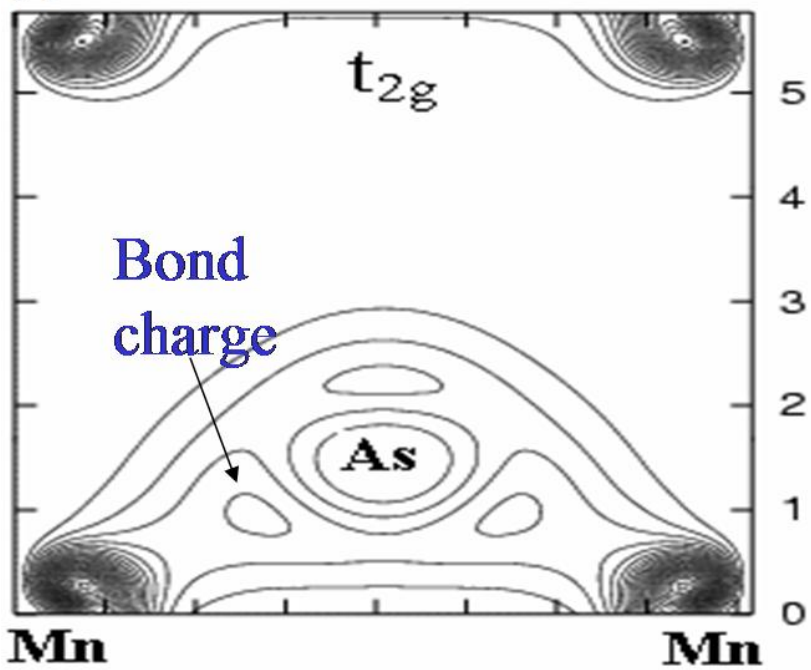

(b)

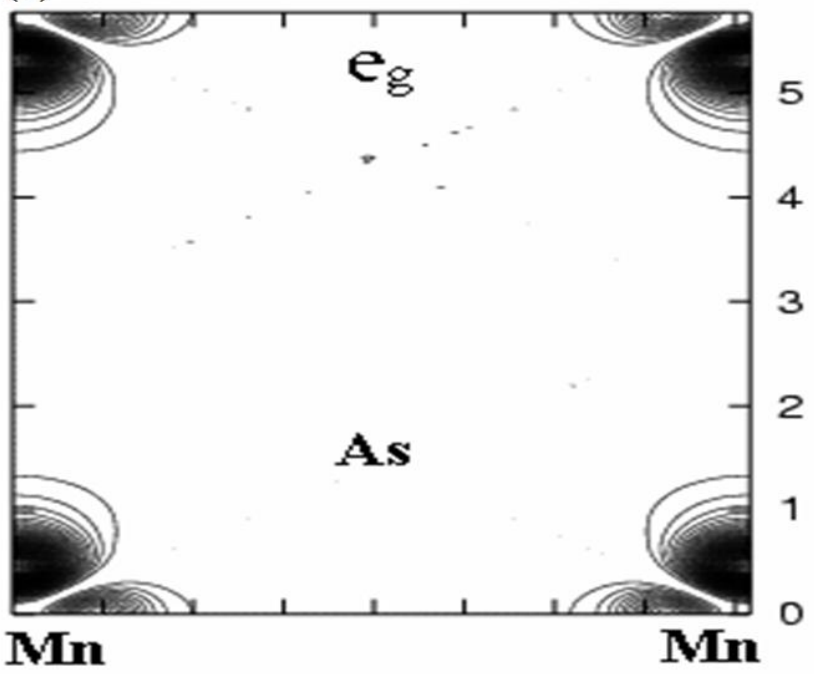


Figure 8. Magnetic moment and total energy vs. lattice constant of zincblende MnC.

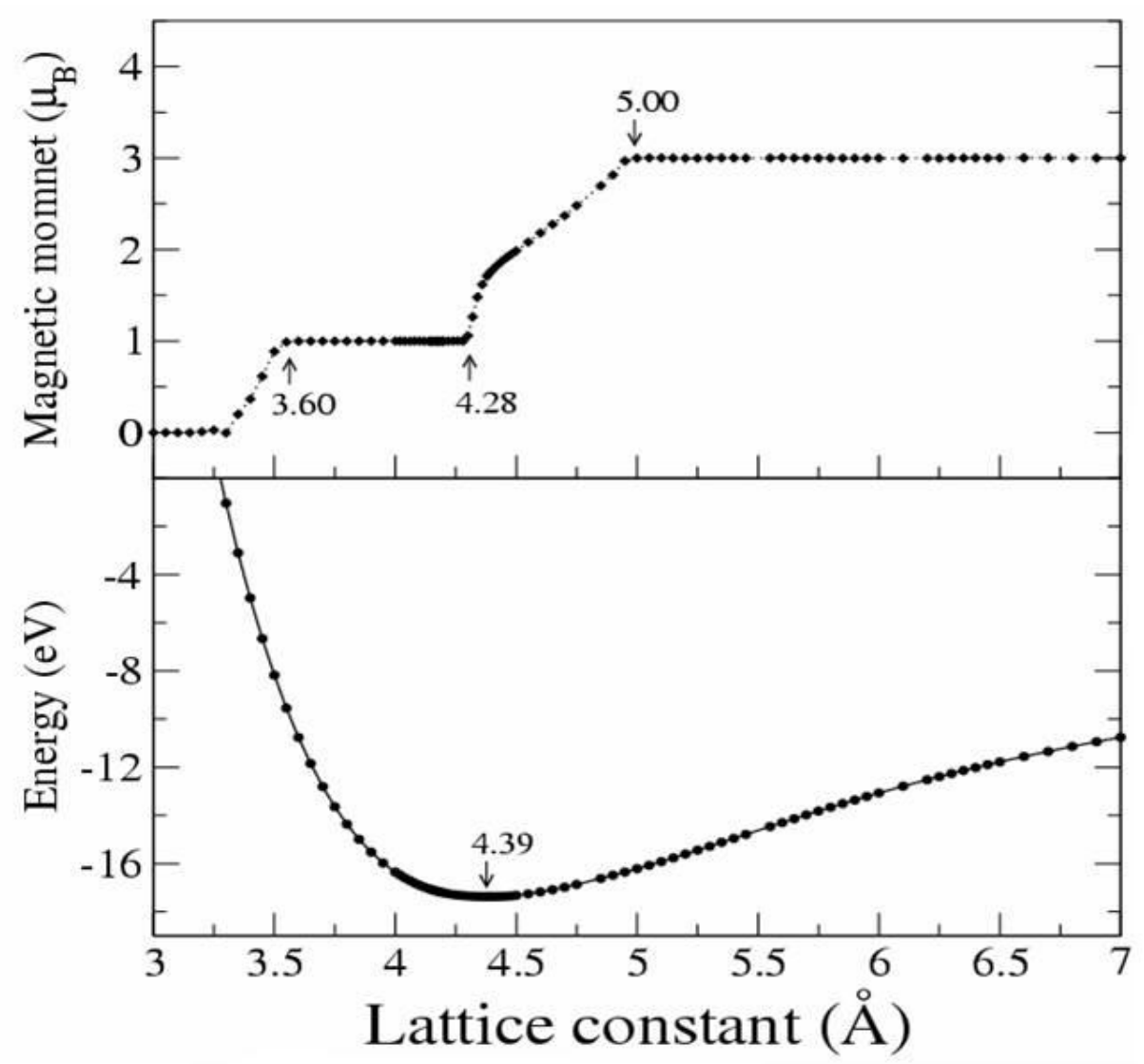


Figure 9. DOS vs. energy of zincblende MnC at $a=4.20 \AA$.

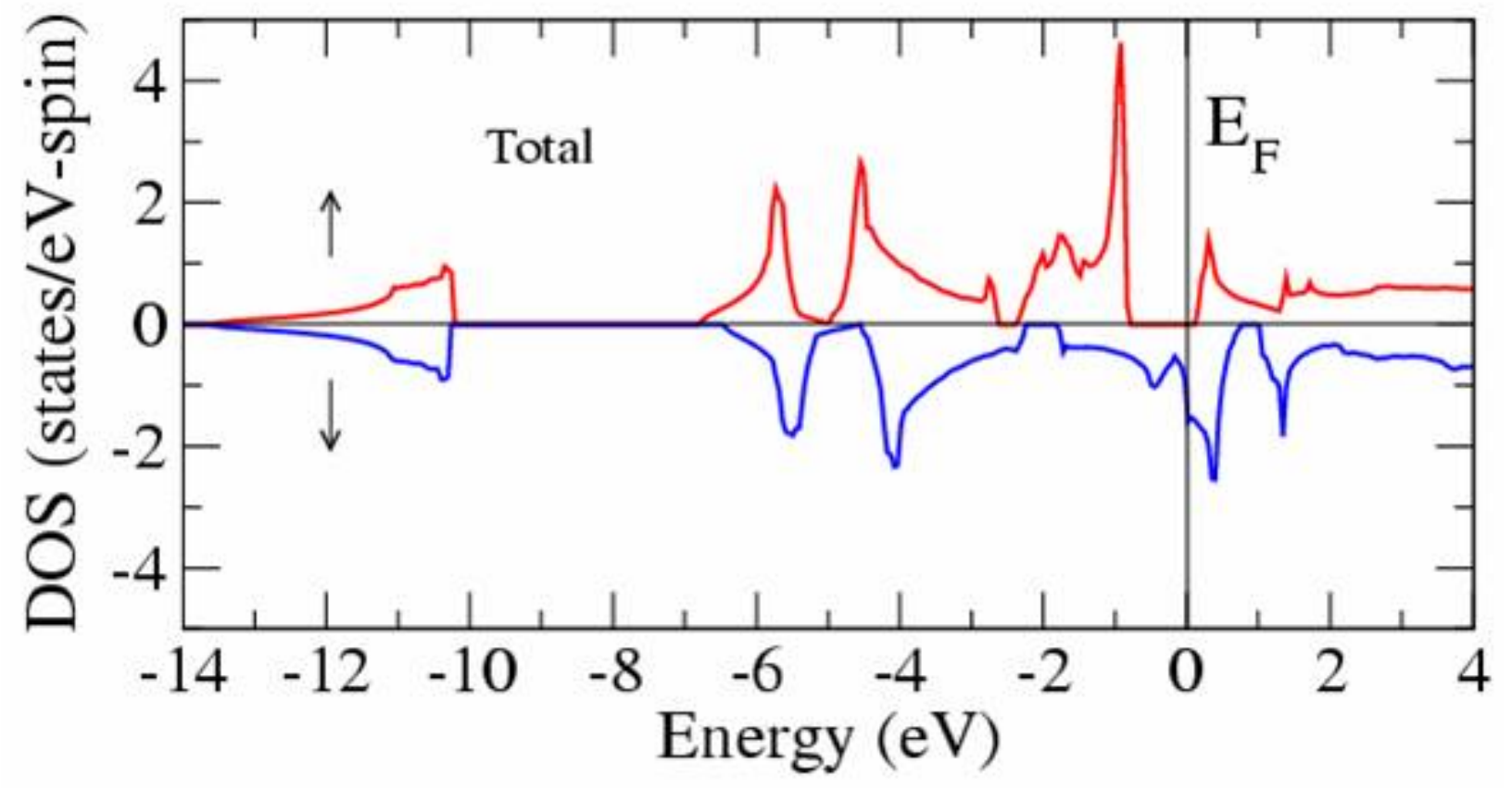


Figure 10. Structure of GaAs/MnAs $/(\mathrm{CrAs})_{2} /(\mathrm{GaAs})_{2}$ superlattice.

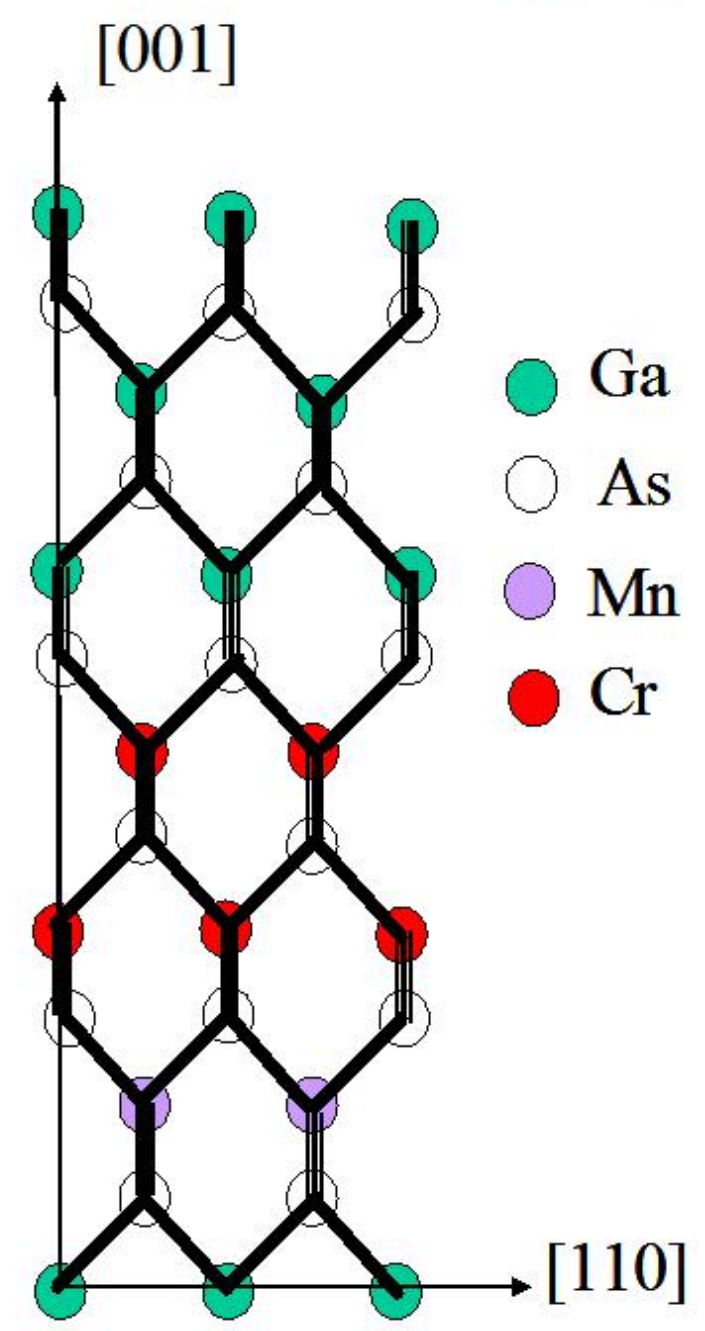


Figure 11. Charge densities in the (0-11) plane, with z-axis along the horizontal, of (a) majority-spin states, (b) minority-spin states, and (c) states with energies within $0.3 \mathrm{eV}$ above $E_{F}$.
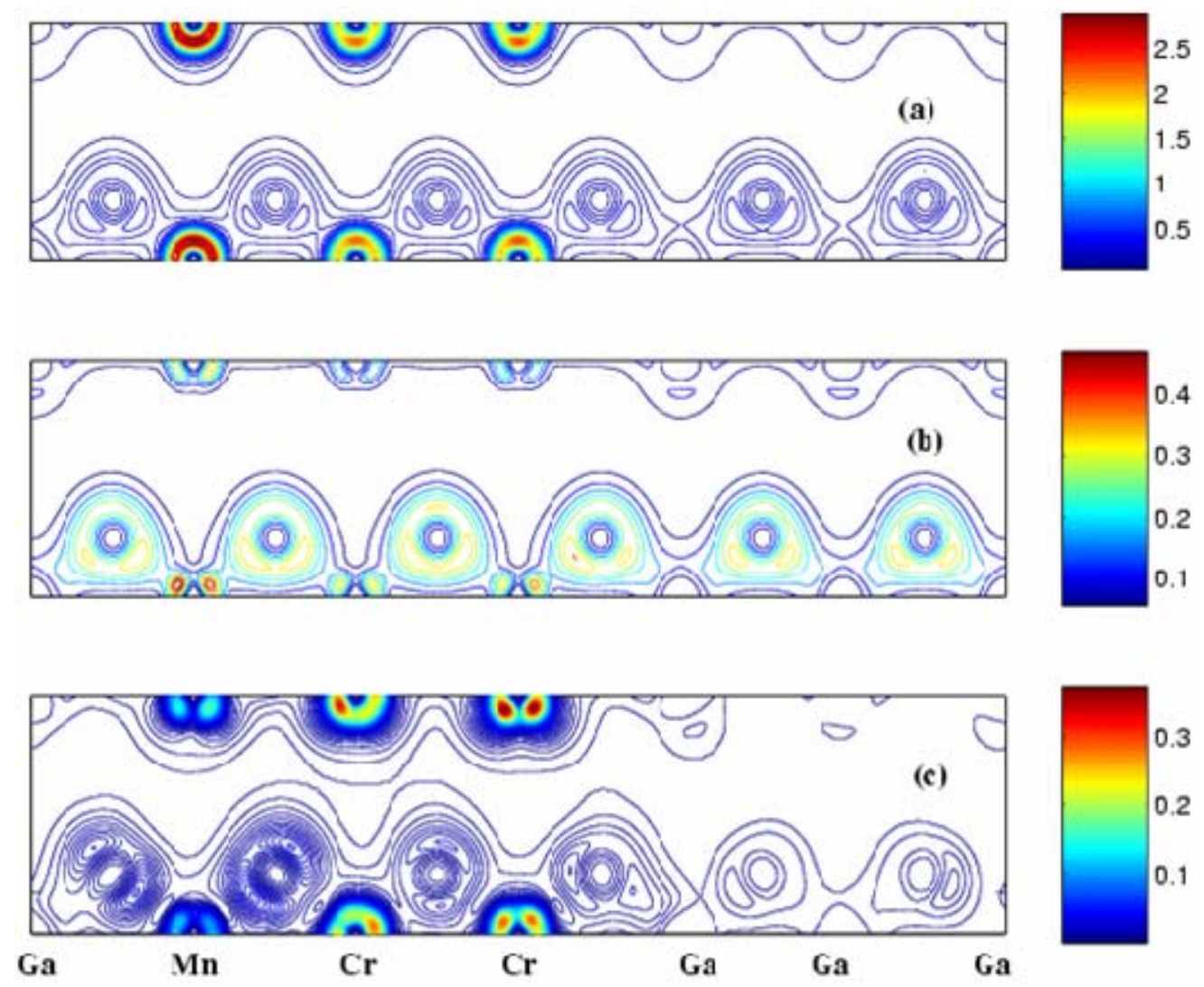
Figure 12. Calculated conductance vs. energy for the $\mathrm{GaAs} / \mathrm{MnAs} /(\mathrm{CrAs})_{2} /(\mathrm{GaAs})_{2}$ superlattice.

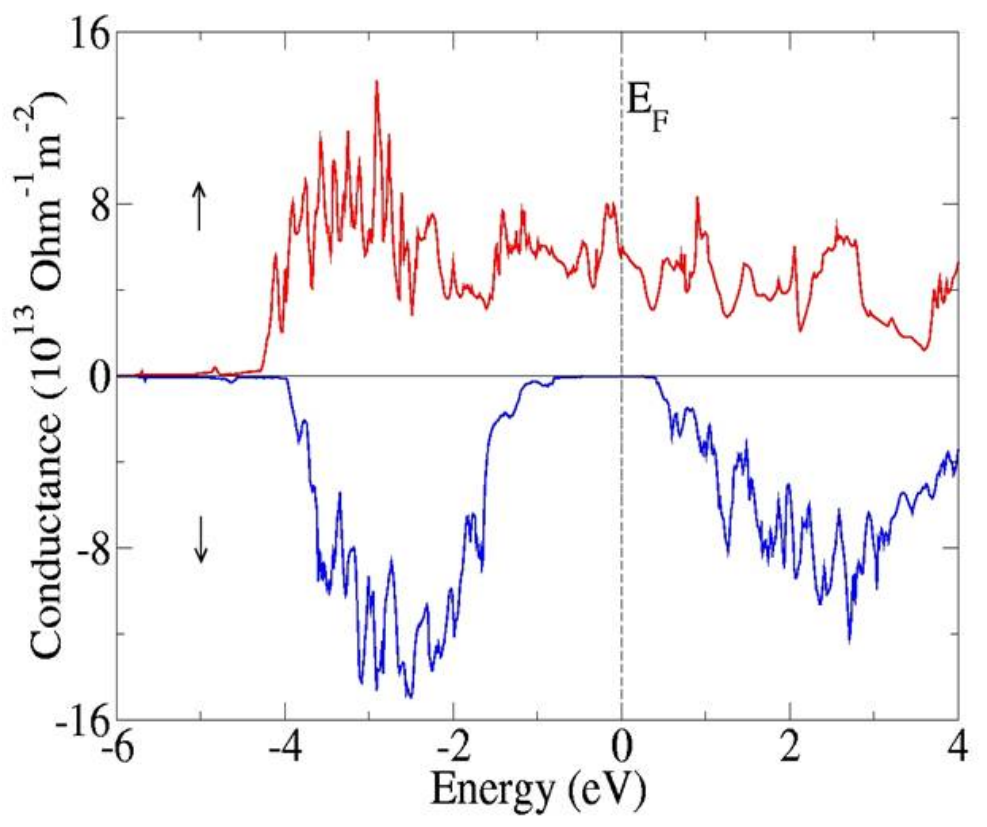


Figure 13. Supercell modeling the $\mathrm{Mn} / \mathrm{Si} \mathrm{DFH}$. The green circles are $\mathrm{Mn}$ atoms, the gray circles are $\mathrm{Si}$.

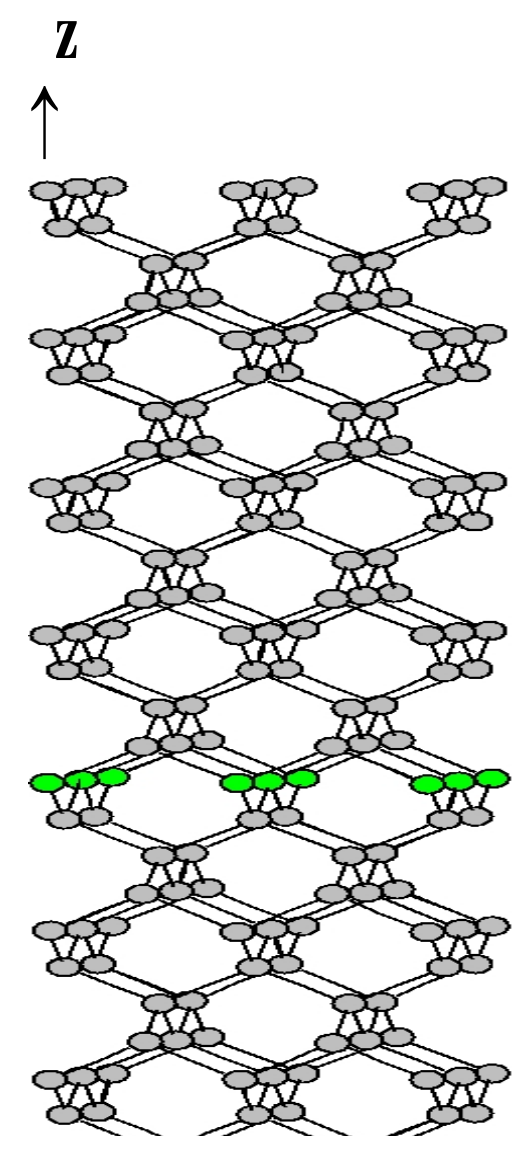


Figure 14. (a) Density of states and (b) two dimensional Fermi surface of the Mn/Si DFH.
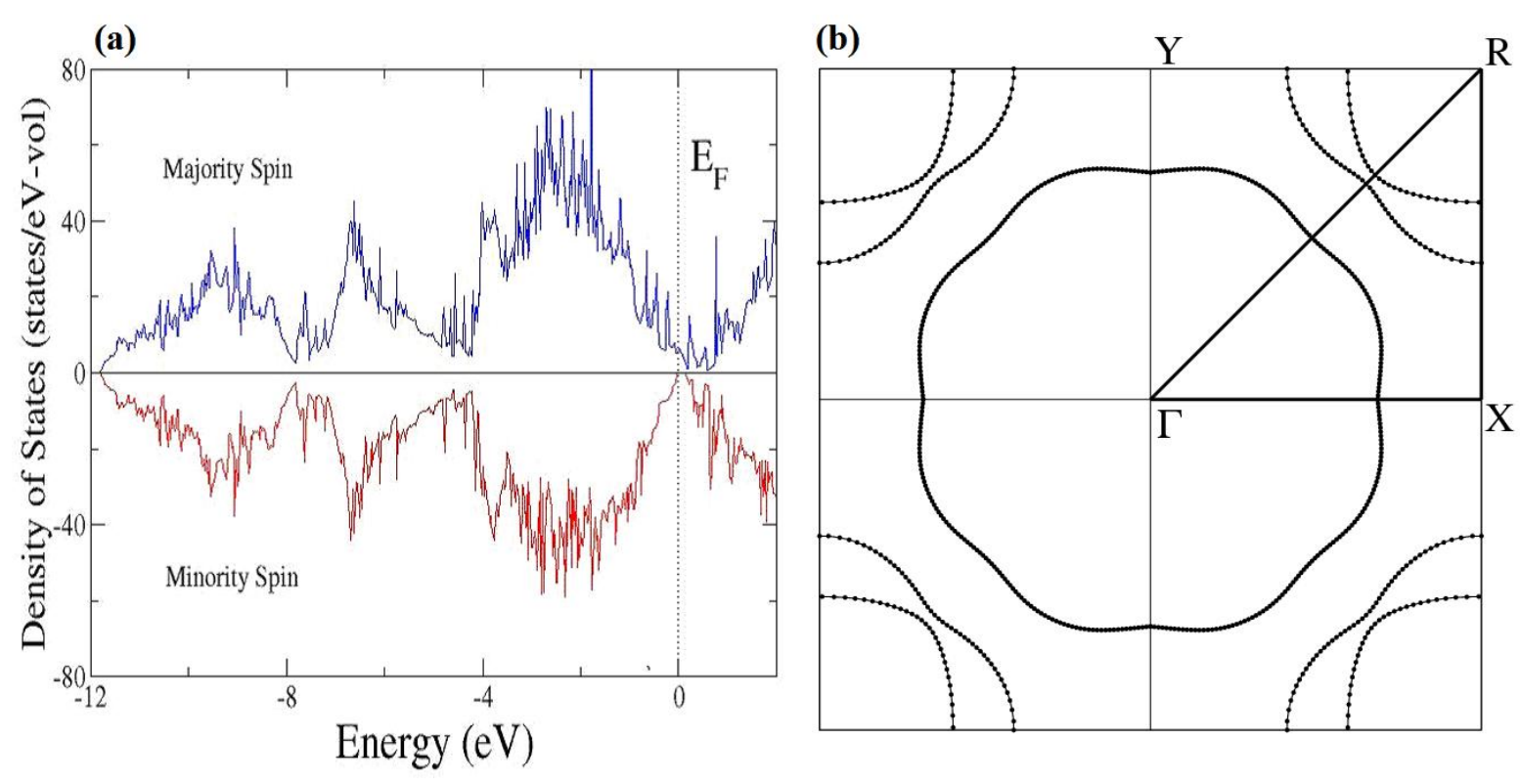
Figure 15. Band structure of (a) majority-spin and (b) minority-spin states of the Mn/Si DFH.
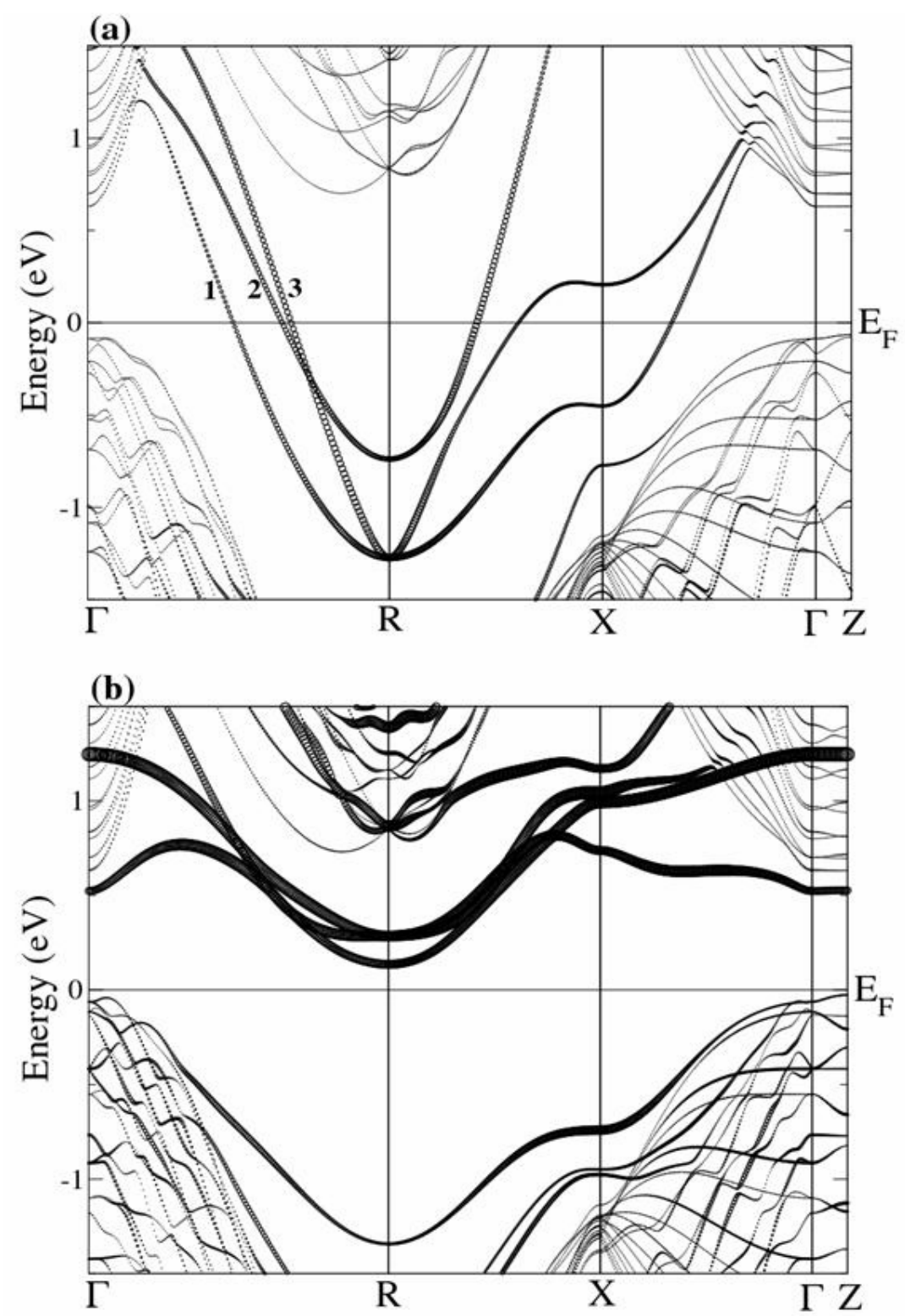
Figure 16. Schematic diagram of the DOS near $E_{F}$ of the Mn/Si DFH.

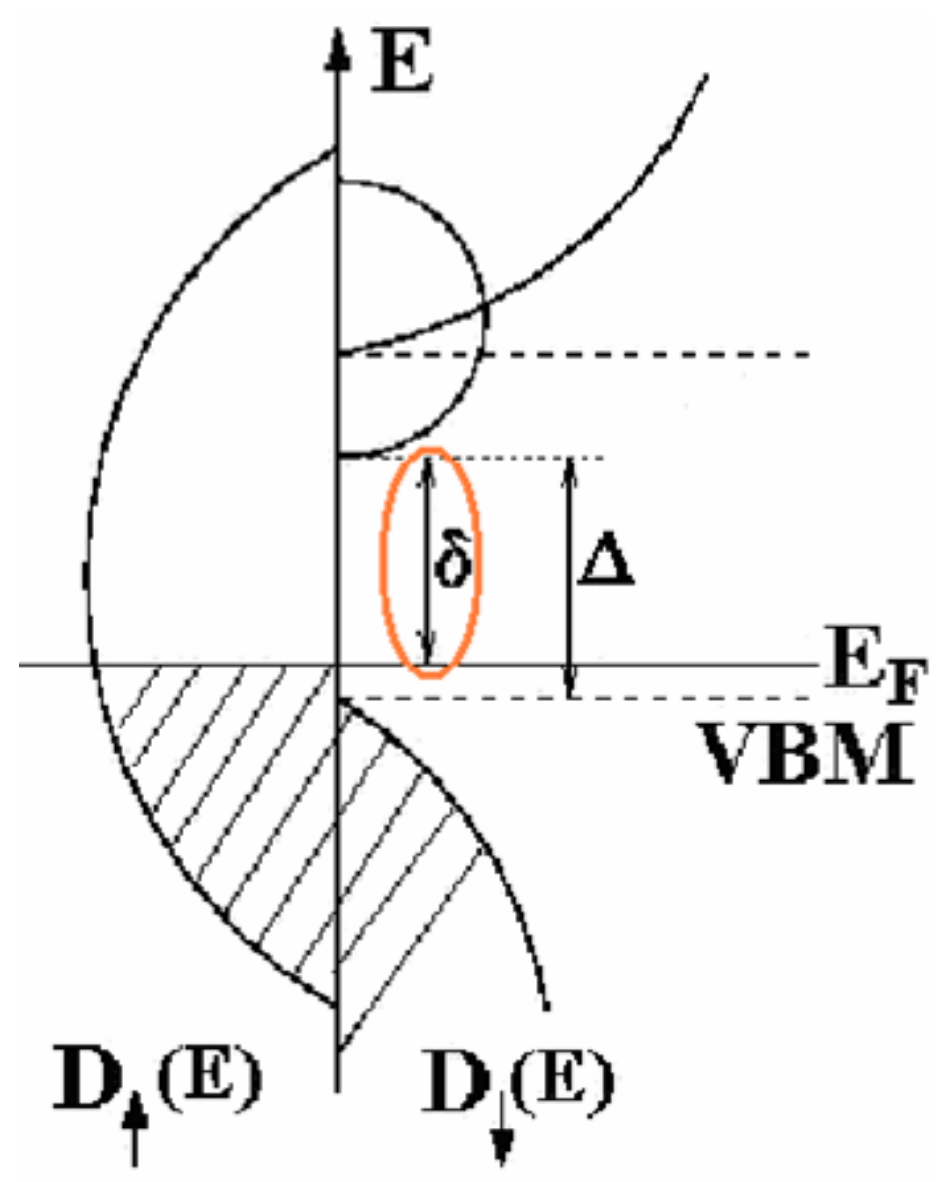

